\begin{tabular}{|c|c|c|}
\hline $\begin{array}{c}\text { PRAMANA } \\
\text { journal of } \\
\text { physics }\end{array}$ & (c) Indian Academy of Sciences & $\begin{array}{l}\text { UWThPh-2004-18 } \\
\text { hep-ph/0407178 } \\
\text { pp. } 1-16\end{array}$ \\
\hline
\end{tabular}

\title{
Physics Possibilities at a Linear Collider*
}

\author{
Alfred Bartl and Stefan Hesselbach \\ Institut für Theoretische Physik, Universität Wien, Boltzmanngasse 5, A-1090 Vienna, Austria
}

\begin{abstract}
We review some recent studies about the parameter determination of top quarks, $W$ bosons, Higgs bosons, supersymmetric particles and in the ADD model of extra dimensions at a linear collider.
\end{abstract}

Keywords. linear collider, standard model, extensions of standard model

PACS Nos 13.66.Fg,13.66.Hk,13.66.Jn,14.65.Ha,14.80.Ly

\section{Introduction}

Up to now the Standard Model (SM) has passed all accelerator-based experimental tests. It is able to reproduce all experimental data obtained at high energy $e^{+} e^{-}, p \bar{p}$ and $e^{ \pm} p$ colliders. In particular, the precision data of LEP have verified the SM predictions with very high accuracy, with the experimental errors being in the range of about $0.1 \%-1 \%$. On the theoretical side, most of the one-loop corrections to the prominent observables have been calculated, in some cases also the leading two-loop corrections are known. The theoretical errors are also in the $0.1 \%-1 \%$ range.

While the gauge sector of the SM is extremely well tested, our theoretical ideas about electroweak symmetry breaking are still not completely convincing. In fact, clarifying the mechanism of electroweak symmetry breaking will be the central problem which we have to solve with the next generation of high energy colliders. In the SM electroweak symmetry breaking is achieved by the Higgs mechanism. The scalar Higgs boson, also predicted by this mechanism, has not been found so far. The mass of the Higgs boson is a free parameter of the theory. If the Higgs mechanism of the SM is correct, then we know already that the mass of the Higgs boson is constrained by the data of LEP: Its lower bound is $m_{H} \gtrsim 114$ $\mathrm{GeV}$ by the direct searches, while from the fits to the precision data an upper bound of $m_{H} \lesssim 250 \mathrm{GeV}$ can be derived. If the mass of the SM Higgs boson is indeed in this range, then there is no doubt that it will be found at LHC.

The Higgs mechanism in its simplest form like in the SM may cause quite severe theoretical problems, namely the so-called "hierarchy problem" and the "fine-tuning problem".

\footnotetext{
* Invited plenary talk presented by A. Bartl at the "Workshop on High Energy Physics Phenomenology (WHEPP-8)”, Indian Institute of Technology, Mumbai, January 5 - 16, 2004.
} 
In order to cure these problems, several new theoretical ideas have been proposed, the most important ones are supersymmetry (SUSY), compositeness, strong electroweak symmetry breaking, extra dimensions etc. In many of these models the Higgs sector is more complicated than in the SM. For example, in the Minimal Supersymmetric Standard Model (MSSM) or in a more general Two-Higgs-Doublet Model the Higgs sector contains two Higgs doublets. The Higgs sector may also contain additional Higgs singlets or may be even more complicated. In all of these cases we will probably see a Higgs state at LHC, however, we may not be able to decide whether it is the Higgs boson of the SM or it is a state of a more complicated Higgs sector.

In some of the different theoretical models which have been proposed as alternatives to the Higgs mechanism, electroweak symmetry breaking is achieved without introducing an elementary scalar Higgs particle. This is the case, for example, in technicolour models or models of strong electroweak symmetry breaking, in models of compositeness and others. All these theoretical ideas have different phenomenological implications which have to be tested in experiment. As has been demonstrated in several recent workshops, an $e^{+} e^{-}$linear collider with a centre-of-mass energy in the range of about $500 \mathrm{GeV}$ to $1 \mathrm{TeV}$ will enable us to distinguish between the different mechanisms of electroweak symmetry breaking and will presumably provide a unique answer to the questions about the origin of mass [1-4].

A new machine like an $e^{+} e^{-}$linear collider must be able to improve our knowledge in two ways: On the one hand it must allow us to discover "new physics", on the other hand it must also provide a better understanding of "known physics" by more precise measurements of the various SM parameters. In the recent workshops [2-4] it has been demonstrated that an $e^{+} e^{-}$linear collider will be a very good discovery machine as well as an excellent precision instrument for physics of and beyond the SM. At a linear collider we will be able to measure the SM parameters like the masses of the top quark and the $W^{ \pm}$boson, the running strong coupling constant $\alpha_{s}$ and many others with much better precision than presently available. This machine will also allow us to determine the parameters of the Higgs and SUSY sectors and other extensions of the SM with very high accuracy. As the signal cross sections decrease with increasing c.m.s. energy, a high luminosity will be required. It is expected that the integrated luminosity will reach a value of approximately $\mathcal{L}=500 \mathrm{fb}^{-1}$ per year of running. Also a high degree of beam polarisation will be necessary [5]. As has been shown, a degree of $80 \%$ and $60 \%$ for the $e^{-}$and $e^{+}$ beam, respectively, can be achieved. Presently there are also discussions about the possible benefits of transverse beam polarisations [6,7]. Furthermore, it is expected that the linear collider can be operated also in the $e^{-} e^{-}, e \gamma$ and $\gamma \gamma$ mode (for reviews see [8]). There is also the proposal to operate the linear collider in the $e^{+} e^{-}$mode at or near $\sqrt{s} \approx m_{Z}$, which is also called the GigaZ mode.

In this talk we will give a selective review of some of the recent studies on the physics possibilities at a linear collider. We will select a few illustrative examples in SM physics and in "new physics" to demonstrate which new results we can expect to gain at such a new machine. For more complete reviews we refer to [1-4]. 


\section{Standard Model Physics}

We have at least two reasons why also in the future we have to perform precision tests of the SM with increasing accuracy:

- We expect that the SM is only an effective theory valid at low energies and we have to find the limits of its validity.

- Usually any "new physics" reaction has a background of SM reactions, which we must know with sufficient precision to extract the signal.

The present situation after the experiments at LEP, SLC and Tevatron can be illustrated by an example from [9] shown in figure 1, which is the result of a global analysis of the electroweak precision data. This figure shows the theoretical relation between the $W^{ \pm}$ mass $m_{W}$ and the top quark mass $m_{t}$ obtained in the SM (red band) and in MSSM (green band) together with the experimental error ellipses. This theoretical relation between $m_{W}$ and $m_{t}$ is due to the radiative corrections to the $W^{ \pm}$boson mass, where the loops involving the top quark play a special role. The leading corrections depend quadratically on $m_{t}$ and logarithmically on the Higgs boson mass $m_{H}$. While in this calculation essentially all basic electroweak parameters enter, $m_{W}$ depends very significantly on $m_{t}$ and on $m_{H}$. The width of the SM band is mainly due to the variation of the Higgs boson mass in the range $113 \mathrm{GeV} \lesssim m_{H} \lesssim 400 \mathrm{GeV}$. The MSSM band is obtained by varying the SUSY parameters in the range allowed by the experimental and theoretical constraints. There is a small overlap of the SM and MSSM bands (blue band) for a light Higgs boson $\left(m_{H}=113 \mathrm{GeV}\right)$ and a heavy SUSY spectrum. As can be seen, the present experimental errors (blue ellipse) do not allow to discriminate between the two models. The data of the linear collider (red ellipse) will presumably allow us to discriminate between the SM and the MSSM or another extension of the SM.

In the calculation of the various observables at the $Z$ resonance the basic electroweak parameters enter, which are the $Z$ boson mass $m_{Z}$, the electromagnetic fine structure constant $\alpha$, the Fermi coupling constant $G_{F}$, and the vector and axial-vector couplings $g_{V}^{f}$ and $g_{A}^{f}$ of the $Z$ boson to the leptons and quarks. $\alpha, G_{F}$ and $m_{Z}$ are known with very high accuracy. The coupling constants $g_{V}^{f}$ and $g_{A}^{f}$ are determined by measurements at the $Z$ resonance, as there are the $Z$ partial decay widths, forward-backward asymmetries, $\tau$ polarisation and its forward-backward asymmetry at LEP, and the left-right asymmetry and the left-right forward-backward asymmetry at SLD [10]. The effective electroweak mixing angle $\sin ^{2} \theta_{\text {eff }}^{f}$ can then be determined via $g_{V}^{f} / g_{A}^{f}=1-4\left|q_{f}\right| \sin ^{2} \theta_{\text {eff }}^{f}$. The weighted average of the experimental result for the leptonic effective electroweak mixing angle is $\sin ^{2} \theta_{\text {eff }}^{\text {lept }}=0.23150 \pm 0.00016$ [10]. In this context it is interesting to note that by operating the linear collider in the GigaZ mode the errors of all the $Z$ couplings could be significantly reduced, leading to an error on the weak mixing angle of approximately $\delta \sin ^{2} \theta_{\text {eff }} \approx \pm 1 \times 10^{-5}$ [11].

Note that in figure 1 for the top quark mass the value $m_{t}=174.3 \pm 5.1 \mathrm{GeV}$ has been used. This value has been changed recently to $m_{t}=178.0 \pm 4.3 \mathrm{GeV}$ [12]. When using this new value for $m_{t}$ the present error ellipse (blue ellipse) will be shifted to the right by approximately $3.7 \mathrm{GeV}$. On the one hand this improves the agreement between the data and the SM predictions, giving $m_{H}=113_{-42}^{+62} \mathrm{GeV}$ for the most probable value of the SM Higgs mass. On the other hand it also shows that, apart from finding the Higgs boson, it 


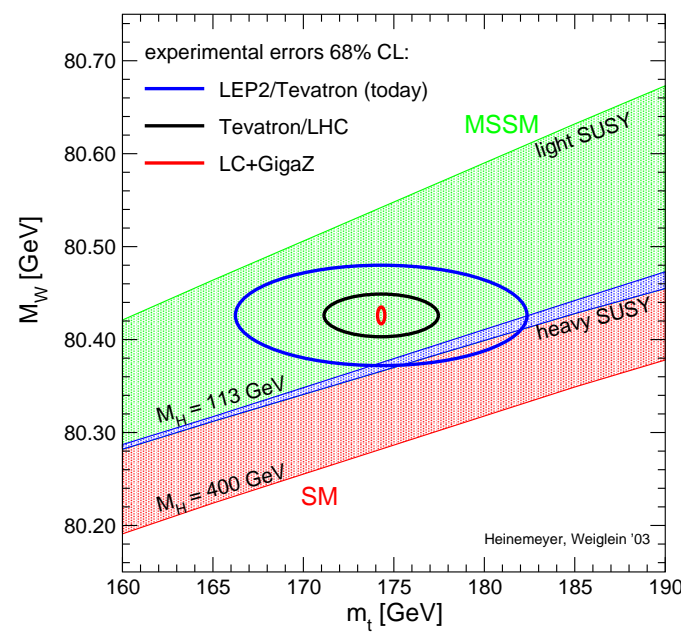

Figure 1. The present experimental accuracy for $m_{W}$ and $m_{t}$ after the experiments at LEP and Tevatron (blue) and the expected accuracies at Tevatron + LHC (black) and LC + GigaZ (red). The red and green bands show the predictions of SM and MSSM, respectively, where the blue band denotes the overlap between the predictions of SM and MSSM. From [9].

would be very desirable to reduce the experimental errors of the masses of the $W^{ \pm}$boson and the top quark. We will shortly review in the next subsections which accuracy for the top quark, $W^{ \pm}$boson and Higgs boson parameters will be obtained at a linear collider.

\subsection{Top Quark Production}

Due to its high mass the top quark plays a special role among the SM particles and it is often claimed that measuring all its parameters very precisely may already open a window to new physics [13]. As has been shown in [14], for example, the precise knowledge of the top quark mass is necessary for the theoretical predictions of electroweak precision observables and for the clarification of several aspects of the Higgs sector and of the MSSM as well as for testing grand unification. At the Tevatron and LHC it is expected that the experimental error of the top quark mass can be reduced to approximately $\delta m_{t}=1-$ $2 \mathrm{GeV}$ (see e.g. [15]). At a linear collider an accuracy for the top quark mass of about $\delta m_{t} \lesssim 100 \mathrm{MeV}$ is envisaged [2-4].

The most precise determination of the top quark mass will be possible by a threshold scan of $e^{+} e^{-} \rightarrow t \bar{t}$ in the region $340 \mathrm{GeV} \lesssim \sqrt{s} \lesssim 380 \mathrm{GeV}$. In order to extract a precise value for $m_{t}$, also theoretical work is necessary. As the top quark width $\Gamma_{t} \approx$ $1.5 \mathrm{GeV}$ is much larger than $\Lambda_{\mathrm{QCD}}$, no toponium bound states are formed, however, their traces may be present in the threshold region. For the theoretical analysis it is necessary to perform a double-expansion of the production cross section $\sigma_{t \bar{t}}$ in the strong coupling $\alpha_{s}$ and the velocity $v$ of the top quark, $v=\sqrt{1-\left(4 m_{t}^{2}\right) / s} \ll 1$. The terms of this expansion up to NNLO have been calculated [16]. There are large logarithms which have been resummed. The error of $m_{t}$ is correlated with that of $\alpha_{s}$, however, a simultaneous 
precision measurement of $m_{t}$ and $\alpha_{s}$ by a threshold scan will be possible. The analyses of $[2-4,16-18]$ indicate that the expected errors are $\delta m_{t}^{\exp +\text { theo }} \approx 100 \mathrm{MeV}, \delta \alpha_{s}\left(M_{Z}\right)^{\exp } \sim$ 0.001 , and for the total width $\delta \Gamma_{t}^{\exp } \sim 50 \mathrm{MeV}$.

Furthermore, by measuring various decay distributions we will obtain information about the top quark couplings. For example, the single decay-lepton angular distributions and angular asymmetries are sensitive to the CP-violating top quark couplings $[19,20]$.

\section{$2.2 W^{ \pm}$Boson Production}

Operating the linear collider near the kinematical threshold of the reaction $e^{+} e^{-} \rightarrow$ $W^{+} W^{-}$will provide a measurement of the $W^{ \pm}$boson mass with an error of $\delta_{m_{W}} \approx$ $\pm 6 \mathrm{MeV}$. Analysing cross sections with polarised beams and various decay distributions will allow us to determine the triple gauge boson couplings with high accuracy.

The most general Lagrangian describing anomalous triple gauge boson couplings is given in [21] and contains 28 real parameters, which can be parameterised by 7 complex coupling parameters $g_{1}^{V}, \kappa_{V}, \lambda_{V}, g_{5}^{V}, g_{4}^{V}, \tilde{\kappa}_{V}$ and $\tilde{\lambda}_{V}$, where $V=\gamma, Z$. The first four couplings are $\mathrm{CP}$ conserving and the last three $\mathrm{CP}$ violating, whereas the real (imaginary) parts of all couplings are CPT conserving (violating), where $\tilde{\mathrm{T}}$ denotes the "naive" time reversal transformation. In the SM all these couplings are zero except $g_{1}^{\gamma}=g_{1}^{Z}=\kappa_{\gamma}=\kappa_{Z}=1$. $g_{1}^{\gamma}$ determines the charge of the $W$ and $\kappa_{\gamma}$, the "anomalous magnetic moment", and $\lambda_{\gamma}$ are related to the magnetic dipole moment and the electric quadrupole moment of the $W$, whereas $\tilde{\kappa}_{\gamma}$ and $\tilde{\lambda}_{\gamma}$ are related to the electric dipole moment and the magnetic quadrupole moment.

In [22] the prospects of measuring the triple gauge couplings at a linear collider with transverse beam polarisation in the process $e^{+} e^{-} \rightarrow W^{+} W^{-}$are studied. The expected errors for the $\mathrm{CP}$ and $\mathrm{CPT}$ conserving couplings are given in table 1 . The sensitivity in the other three (CP, CPT) symmetry classes is comparable and there is no statistical correlation between the classes. Within a symmetry class there are large statistical correlations. Without beam polarisation 27 coupling parameters appear to be measurable, longitudinal $e^{-}$beam polarisation $P_{e^{-}}$improves the sensitivity by a factor $\sim 2$, additional longitudinal $e^{+}$beam polarisation $P_{e^{+}}$by a factor $3-4$. With transverse beam polarisation $\left(P_{e^{-}}^{t}, P_{e^{+}}^{t}\right)$ all couplings may be accessible at a linear collider and there is a factor $2-4$ improvement in comparison to unpolarised beams. The couplings $\kappa_{\gamma}$ and $\lambda_{\gamma}$ have also been analysed at a photon collider [23]. With help of the process $e^{-} \gamma \rightarrow W^{-} \nu$ it is possible to improve the sensitivity on $\lambda_{\gamma}$ by a factor 1.5 in comparison to the process $e^{+} e^{-} \rightarrow W^{+} W^{-}$with both beams polarised, whereas the sensitivity on $\kappa_{\gamma}$ is of the same order of magnitude.

\subsection{Higgs Boson Production}

The search for the Higgs boson will have a very high priority at LHC as well as at the linear collider [2-4,24]. The production of a SM Higgs boson in $e^{+} e^{-}$annihilation can proceed via "Higgsstrahlung" $e^{+} e^{-} \rightarrow Z H, W W$ fusion $e^{+} e^{-} \rightarrow \nu_{e} \bar{\nu}_{e} H$, and $Z Z$ fusion $e^{+} e^{-} \rightarrow e^{+} e^{-} H$. The relative importance of Higgsstrahlung and $W W$ fusion is shown in figure 2 (from [3,24]). At $\sqrt{s}=500 \mathrm{GeV}$ the Higgsstrahlung process dominates for $m_{H} \gtrsim 160 \mathrm{GeV}$, whereas for $m_{H} \lesssim 160 \mathrm{GeV}$ the $W W$ fusion process gives the largest 
Table 1. Expected errors in units of $10^{-3}$ on the $\mathrm{CP}$ and CPT̃ conserving couplings in the presence of all anomalous couplings at $\sqrt{s}=500 \mathrm{GeV}$, with unpolarised beams and with different beam polarisations. From [22].

\begin{tabular}{l|ccccccccc}
\hline & $\operatorname{Re} \Delta g_{1}^{\gamma}$ & $\operatorname{Re} \Delta g_{1}^{Z}$ & $\operatorname{Re} \Delta \kappa_{\gamma}$ & $\operatorname{Re} \Delta \kappa_{Z}$ & $\operatorname{Re} \lambda_{\gamma}$ & $\operatorname{Re} \lambda_{Z}$ & $\operatorname{Re} g_{5}^{\gamma}$ & $\operatorname{Re} g_{5}^{Z}$ \\
\hline Unpolarised beams & 6.5 & 5.2 & 1.3 & 1.4 & 2.3 & 1.8 & 4.4 & 3.3 \\
$\left(P_{e^{-}}, P_{e^{+}}\right)=(\mp 80 \%, 0)$ & 3.2 & 2.6 & 0.61 & 0.58 & 1.1 & 0.86 & 2.2 & 1.7 \\
$\left(P_{e^{-}}, P_{e^{+}}\right)=(\mp 80 \%, \pm 60 \%)$ & 1.9 & 1.6 & 0.40 & 0.36 & 0.62 & 0.50 & 1.4 & 1.1 \\
$\left(P_{e^{-}}^{t}, P_{e^{+}}^{t}\right)=(80 \%, 60 \%)$ & 2.8 & 2.4 & 0.69 & 0.82 & 0.69 & 0.55 & 2.5 & 1.9 \\
\hline
\end{tabular}

contribution. The higher $\sqrt{s}$ the more important is the $W W$ fusion process. For $\sqrt{s} \gtrsim$ $800 \mathrm{GeV}$ the $Z Z$ fusion process can contribute about $\gtrsim 10 \%$ of the total production rate. As can also be seen in figure 2 , if $m_{H} \lesssim 250 \mathrm{GeV}$ as suggested by the electroweak precision data, an optimal choice for the c.m.s. energy is $\sqrt{s} \approx 350-500 \mathrm{GeV}$.

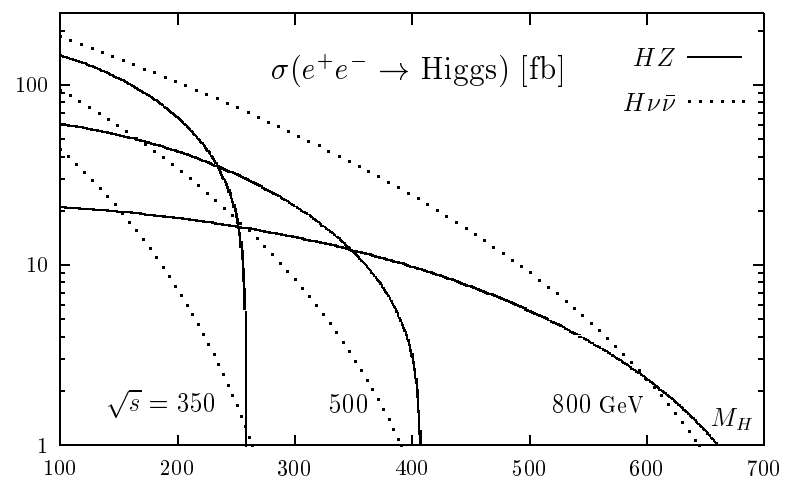

Figure 2. The Higgsstrahlung and $W W$ fusion production cross sections versus the Higgs mass for $\sqrt{s}=300 \mathrm{GeV}, 500 \mathrm{GeV}$ and $800 \mathrm{GeV}$. From [3,24].

In order to distinguish a SM Higgs boson from a state of a more complicated Higgs sector it will be necessary to measure its decay branching ratios with good precision. The couplings of the SM Higgs to the fermions and vector bosons are proportional to their masses. Hence, if the Higgs boson mass is fixed, then also all decay widths are known. In figure 3 from $[3,24]$ we show the most important branching ratios of the SM Higgs and the total width as a function of its mass. As can be seen, beyond the threshold of the decay $H \rightarrow W^{+} W^{-}$the width rises rather strongly with the mass. For $m_{H}<150 \mathrm{GeV}$ the decay $H \rightarrow b \bar{b}$ dominates, whereas the branching ratios for $H \rightarrow \tau^{+} \tau^{-}, H \rightarrow c \bar{c}$ and $H \rightarrow g g$ are of the order of a few percent. It is expected that for a light Higgs boson the coupling to the bottom quark can be determined with an error of about $2 \%$ and those to the charm quark and the $\tau$ lepton with about $10 \%$. The coupling of the SM Higgs boson to the top quark is particularly interesting. For $m_{H}<2 m_{t}$ a possible way to determine this coupling is given by measuring the associated production process $e^{+} e^{-} \rightarrow t \bar{t} H$. For measuring this coupling with an error of about $10 \%$ we will need higher energy, $\sqrt{s} \gtrsim 800 \mathrm{GeV}$, and higher luminosity, $\mathcal{L} \approx 1000 \mathrm{fb}^{-1}$ per one year of running. 

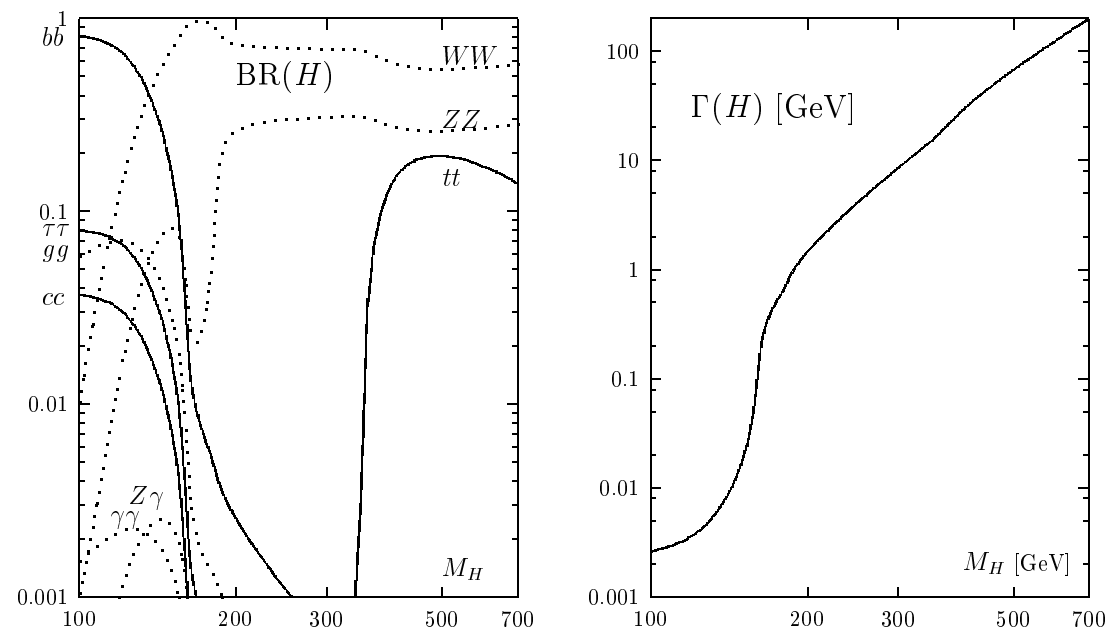

Figure 3. (a) Branching ratios and (b) the total decay width of the SM Higgs boson as a function of its mass. From [3,24].

A further test for the scalar potential of the SM Higgs boson will be provided by measurements of its self-couplings. The 3-Higgs coupling is given by $\lambda_{3}=m_{H}^{2} /(2 v)$ and the 4-Higgs coupling by $\lambda_{4}=m_{H}^{2} /\left(2 v^{2}\right)$, where $v=246 \mathrm{GeV}$ is the vacuum expectation value of the Higgs field after spontaneous electroweak symmetry breaking. The coupling $\lambda_{3}$ can be measured in the "double-Higgsstrahlung" process $e^{+} e^{-} \rightarrow Z H H$. The analyses of $[25,26]$ have shown that $\lambda_{3}$ can be measured with an error of approximately $20 \%$ in the Higgs mass range $120 \mathrm{GeV}<m_{H}<140 \mathrm{GeV}$ with an integrated luminosity of $1000 \mathrm{fb}^{-1}$. The 4-Higgs coupling $\lambda_{4}$ can in principle be measured in the "triple-Higgsstrahlung" process, which however is suppressed [26].

It is also necessary to verify that the Higgs boson is a spin $0 \mathrm{CP}$ even particle. At the linear collider this can be done in several ways: (i) By measuring the angular dependence of $e^{+} e^{-} \rightarrow Z H$, which must be $d \sigma / d \cos \theta \propto \sin ^{2} \theta$ for a $0^{+}$particle. (ii) By measuring the $\sqrt{s}$ dependence of $e^{+} e^{-} \rightarrow Z H$ near threshold. (iii) If $m_{H}<2 m_{Z}$ by measuring the angular dependence of the decay products in the decay $H \rightarrow Z Z^{*}$ and by measuring the invariant mass distribution of the virtual $Z$.

\section{Beyond the standard model}

\subsection{Extended Higgs sectors}

In models beyond the SM additional Higgs doublet and singlet fields can occur. In the TwoHiggs-Doublet Models, the simplest of these extended models, the Higgs sector consists of two doublet fields, which generate five physical Higgs particles: the CP-even $h$ and $H$, the CP-odd $A$ and the charged $H^{ \pm}$. In particular the Higgs sector of the Minimal Supersymmetric Standard Model (MSSM) contains two doublets, which are necessary to break the electroweak symmetry. 
Supersymmetry leads to several relations among the parameters of the MSSM Higgs sector resulting at tree-level in only two independent parameters and mass relations like $m_{h} \leq m_{Z}, m_{A} \leq m_{H} ; m_{H^{ \pm}} \geq m_{W} ; m_{H^{ \pm}}^{2}=m_{A}^{2}+m_{W}^{2} ; m_{h}^{2}+m_{H}^{2}=m_{A}^{2}+m_{Z}^{2}$. Radiative corrections, however, break some of these correlations and especially $m_{h}$ is pushed upwards by several ten $\mathrm{GeV}$. A recent analysis, including full one-loop corrections and the two-loop corrections controlled by $\alpha_{s}$ and the Yukawa couplings of the third generation fermions and using the latest value of the top quark mass [12], gives a conservative upper bound $m_{h} \lesssim 152 \mathrm{GeV}$ [27].

In the two-Higgs-doublet extensions of the SM the CP-even Higgs bosons $h$ and $H$ can be produced by the associated production process $e^{+} e^{-} \rightarrow A+h, H$ in addition to Higgsstrahlung and $W W$ fusion. The CP-odd Higgs boson $A$ cannot be produced in Higgsstrahlung and fusion processes at leading order. The charged Higgs bosons $H^{ \pm}$can be directly pair produced, $e^{+} e^{-} \rightarrow H^{+} H^{-}$. Figure 4 from $[3,24]$ shows representative examples of $e^{+} e^{-} \rightarrow Z+h, H$ and $e^{+} e^{-} \rightarrow A+h, H$ production cross sections in the MSSM as a function of the respective Higgs masses.

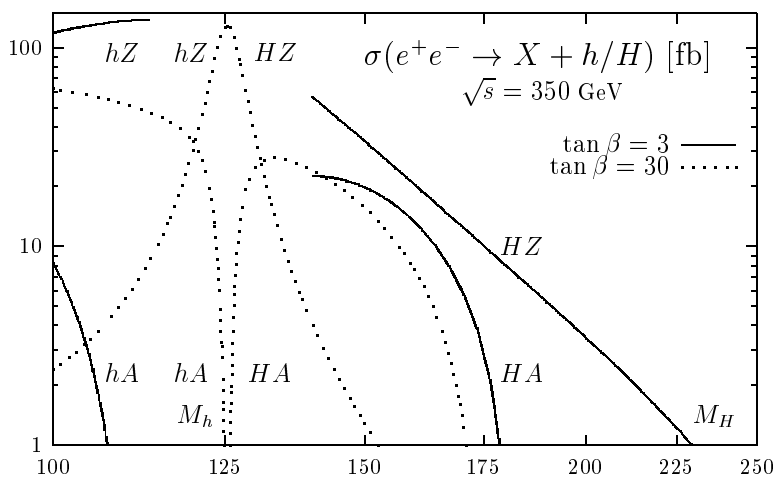

Figure 4. Production cross sections of the neutral Higgs bosons in the MSSM at $\sqrt{s}=350 \mathrm{GeV}$ in the Higgsstrahlung and pair production processes for $\tan \beta=3$ and 30. From $[3,24]$.

In the CP-violating MSSM with complex parameters the CP invariance of the Higgs potential at tree-level is explicitely broken by loop effects of the Yukawa interactions of the third generation squarks [28]. This results in a mixing of the neutral Higgs states $h, H$ and $A$ into three mass eigenstates $H_{1}, H_{2}$ and $H_{3}$. This mixing can considerably change the mass spectrum and couplings of the neutral Higgs bosons and has to be taken into account when analysing the complex MSSM.

\subsection{Supersymmetry}

One of the main goals of a future $e^{+} e^{-}$linear collider will be the search for supersymmetric (SUSY) particles [1-4]. The neutralinos, the supersymmetric partners of the neutral gauge and Higgs bosons, will be particularly interesting because they are relatively light. The lightest neutralino $\tilde{\chi}_{1}^{0}$ is expected to be the lightest SUSY particle (LSP), which is stable if $R$-parity is conserved and the second lightest neutralino $\tilde{\chi}_{2}^{0}$ will presumably be 
among the lightest visible SUSY particles. Therefore, the study of production and decay of the neutralinos $\tilde{\chi}_{i}^{0}, i=1, \ldots, 4,[1-4,29]$ and a precise determination of the underlying supersymmetric parameters $M_{1}, M_{2}, \mu$ and $\tan \beta$ including the phases $\phi_{M_{1}}$ and $\phi_{\mu}$ of $M_{1}$ and $\mu$ will play an important role at future linear colliders. In [30] methods to determine these parameters based on neutralino and chargino mass and cross section measurements have been presented.

The production of neutralinos $e^{+} e^{-} \rightarrow \tilde{\chi}_{i}^{0} \tilde{\chi}_{j}^{0}, i, j=1, \ldots 4$, at a linear collider with polarised beams with subsequent leptonic tree-body decays $\tilde{\chi}_{i}^{0} \rightarrow \tilde{\chi}_{k}^{0} \ell^{+} \ell^{-}$and $\tilde{\chi}_{j}^{0} \rightarrow \tilde{\chi}_{l}^{0} \ell^{+} \ell^{-}$, is analysed in [31]. Since observables like decay angular distributions and T-odd triple product correlations depend on the polarisation of the decaying neutralinos [32] the full spin correlations between production and decay are included. In [31] the complete analytical formulae for longitudinal polarised beams are given, including complex couplings to allow the study of $\mathrm{CP}$ violating phenomena. In figure 5 the cross sections $\sigma\left(e^{+} e^{-} \rightarrow \tilde{\chi}_{1}^{0} \tilde{\chi}_{2}^{0}\right)$ and $\sigma\left(e^{+} e^{-} \rightarrow \tilde{\chi}_{2}^{0} \tilde{\chi}_{2}^{0}\right)$ in the scenario SPS1a [33] are shown as a function of the $e^{+} e^{-}$centre of mass energy $\sqrt{s}$ for several beam polarisations.
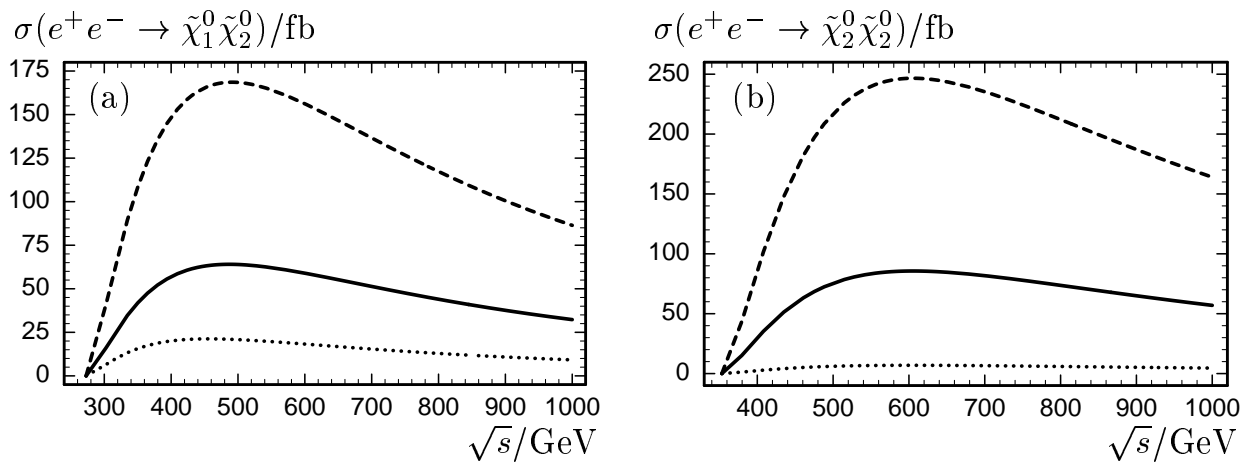

Figure 5. Cross sections for (a) $e^{+} e^{-} \rightarrow \tilde{\chi}_{1}^{0} \tilde{\chi}_{2}^{0}$ and (b) $e^{+} e^{-} \rightarrow \tilde{\chi}_{2}^{0} \tilde{\chi}_{2}^{0}$ in the scenario SPS1a [33] for unpolarised beams (solid) and beam polarisations $P_{e^{-}}=-0.8$, $P_{e^{+}}=+0.6$ (dashed), $P_{e^{-}}=+0.8, P_{e^{+}}=-0.6$ (dotted).

The pair production of light sleptons $e^{+} e^{-} \rightarrow \tilde{\ell}^{+} \tilde{\ell}^{-}, \tilde{\ell}=\tilde{e}_{R}, \tilde{\mu}_{R}, \tilde{\tau}_{1}$, allows to determine the masses of the sleptons with high accuracy from the energy distributions of the final state particles in the slepton decays $\tilde{\ell} \rightarrow \ell \tilde{\chi}_{1}^{0}$. The masses of $\tilde{e}_{R}$ and $\tilde{\mu}_{R}$ can be determined from the energy spectrum of the final electron and muon, respectively and $m_{\tilde{\tau}_{1}}$ from the energy spectra $E_{\rho}$ and $E_{3 \pi}$ from the $\tau$ decays $\tau \rightarrow \rho \nu_{\tau}$ and $\tau \rightarrow 3 \pi \nu_{\tau}$. The study in [38] for the TESLA linear collider [3] in the scenario SPS1a [33] expects the accuracies $m_{\tilde{e}_{R}}=(142.99 \pm 0.08) \mathrm{GeV}, m_{\tilde{\mu}_{R}}=(143.15 \pm 0.17) \mathrm{GeV}$ and $m_{\tilde{\tau}_{1}}=(133.2 \pm 0.3) \mathrm{GeV}$ for $\sqrt{s}=400 \mathrm{GeV}$, beam polarisations $P_{e^{-}}=+0.8, P_{e^{+}}=-0.6$ and an integrated luminosity $\mathcal{L}=200 \mathrm{fb}^{-1}$.

Whereas the parameters $M_{1}, M_{2}$ and $\mu$ including the phases $\phi_{M_{1}}$ and $\phi_{\mu}$ can be determined by measurements of CP-even [30] and CP-odd [34-37] observables in the neutralino and chargino sector, it is more difficult to measure the trilinear couplings $A_{f}$ in the sfermion sector. Cross section measurements of sfermion production processes allow the determination of the sfermion masses and mixing angles (see figure 6) which in turn allow the determination of the parameters $A_{f}$ [39].

In $[40,41]$ production and decays of the third generation sfermions in the MSSM with 

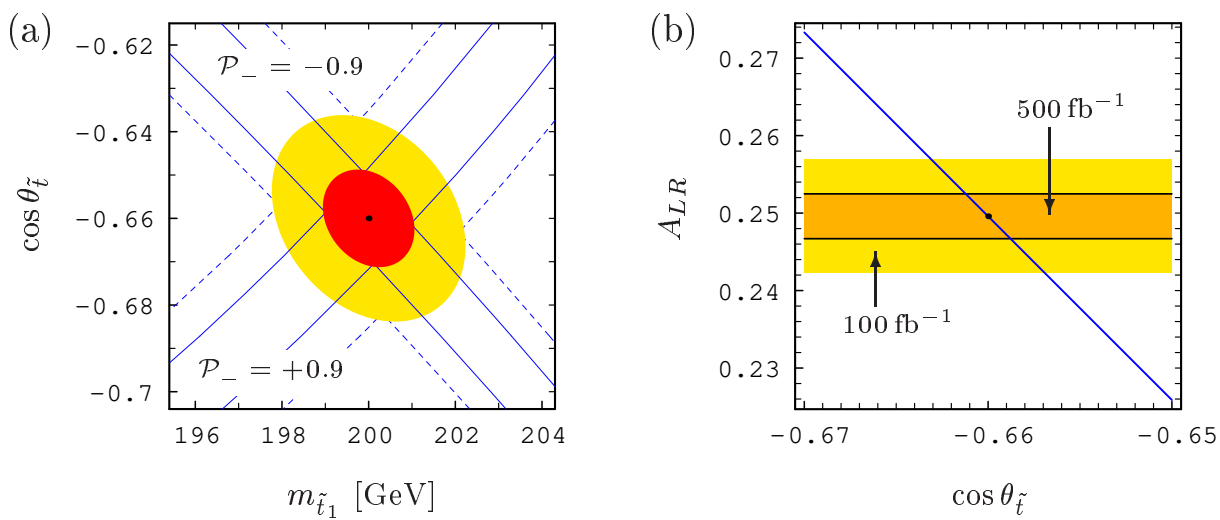

Figure 6. (a) Error bands and $68 \% \mathrm{CL}$ error ellipse for determining the $\tilde{t}_{1}$ mass $m_{\tilde{t}_{1}}$ and mixing angle $\cos \theta_{\tilde{t}}$ from cross section measurements; the dashed lines are for $\mathcal{L}=100 \mathrm{fb}^{-1}$ and the full lines for $\mathcal{L}=500 \mathrm{fb}^{-1}$. (b) Error bands for the determination of $\cos \theta_{\tilde{t}}$ from the left-right asymmetry $A_{L R}$. In both plots $m_{\tilde{t}_{1}}=200 \mathrm{GeV}$, $\cos \theta_{\tilde{t}}=-0.66, \sqrt{s}=500 \mathrm{GeV}, P_{e^{-}}= \pm 0.9, P_{e^{+}}=0$. From [39].

complex parameters $A_{\tau}, A_{t}, A_{b}, \mu$ and $M_{1}$ have been analysed. In a large region of the MSSM parameter space the branching ratios of $\tilde{\tau}_{1,2}, \tilde{\nu}_{\tau}, \tilde{t}_{1,2}$ and $\tilde{b}_{1,2}$ show a strong phase dependence. This could have an important impact on the search for third generation sfermions at a future linear collider and on the determination of the supersymmetric parameters.

In [40] the effects of the CP phases of $A_{\tau}, \mu$ and $M_{1}$ on production and decay of $\tilde{\tau}_{1,2}$ and $\tilde{\nu}_{\tau}$ have been studied. The branching ratios of fermionic decays of $\tilde{\tau}_{1}$ and $\tilde{\nu}_{\tau}$ show a significant phase dependence for $\tan \beta \lesssim 10$ whereas it becomes less pronounced for $\tan \beta>10$. The branching ratios of the $\tilde{\tau}_{2}$ into Higgs bosons depend very sensitively on the phases for $\tan \beta \gtrsim 10$.

In [41] the impact of the CP phases of $A_{t}, A_{b}, \mu$ and $M_{1}$ on the decays of $\tilde{t}_{1,2}$ and $\tilde{b}_{1,2}$ has been analysed. The branching ratios of the $\tilde{t}_{1,2}$ show a pronounced phase dependence in a large region of the MSSM parameter space (figure 7). In the case of $\tilde{b}_{i}$ decays there can be an appreciable $\varphi_{A_{b}}$ dependence, if $\tan \beta$ is large and the decays into Higgs bosons are allowed.

Further the expected accuracy in determining the supersymmetric parameters has been estimated by a global fit of measured masses, branching ratios and production cross sections. $A_{\tau}, A_{t}$ and $A_{b}$ can be expected to be measured with $10 \%, 2-3 \%$ and $50 \%$ accuracy, respectively, $\tan \beta$ with $1 \%$ (2\%) accuracy in case of small (large) $\tan \beta$ and the other parameters with approximately $1 \%$ accuracy.

The fundamental parameters at the GUT or unification scale in specific SUSY breaking models can be reconstructed by evolution of the measured parameters at the electroweak scale to the high scale with help of the respective renormalization group equations (RGE). In [42] the evolution in minimal supergravity (mSUGRA), in left-right supergravity, in gauge mediated SUSY breaking and in superstring induced SUSY breaking models is analysed and the RGE are given. In figure 8 from [43] the evolution of the gaugino mass parameters and of the first generation sfermion mass parameters is shown in the mSUGRA scenario SPS1a [33], where the experimental errors at the electroweak scale 

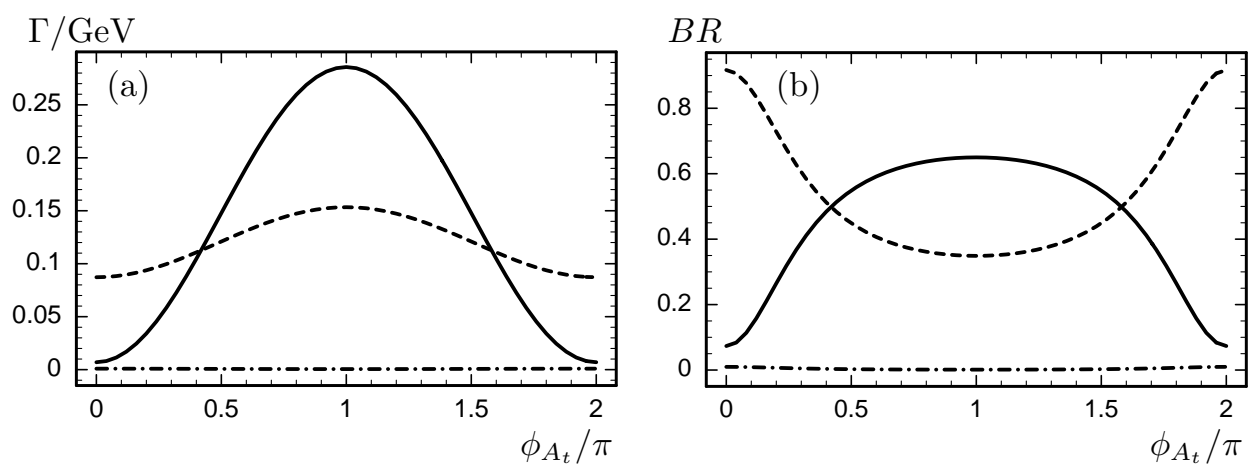

Figure 7. (a) Partial decay widths $\Gamma$ and (b) branching ratios $B R$ of the decays $\tilde{t}_{1} \rightarrow \tilde{\chi}_{1}^{+} b$ (solid), $\tilde{t}_{1} \rightarrow \tilde{\chi}_{1}^{0} t$ (dashed) and $\tilde{t}_{1} \rightarrow W^{+} \tilde{b}_{1}$ (dashdotted) for $\tan \beta=6$, $M_{2}=300 \mathrm{GeV}, M_{1} / M_{2}=5 / 3 \tan ^{2} \theta_{W},|\mu|=350 \mathrm{GeV},\left|A_{b}\right|=\left|A_{t}\right|=800 \mathrm{GeV}$, $\varphi_{\mu}=\pi, \varphi_{M_{1}}=\varphi_{A_{b}}=0, m_{\tilde{t}_{1}}=350 \mathrm{GeV}, m_{\tilde{t}_{2}}=700 \mathrm{GeV}, m_{\tilde{b}_{1}}=170 \mathrm{GeV}$, $M_{\tilde{Q}}>M_{\tilde{U}}$ and $m_{H^{ \pm}}=900 \mathrm{GeV}$. From [41].

have been fixed by a coherent combination of the analyses for the LHC and for a linear collider. This allows to determine the mSUGRA parameters with the following accuracies: $m_{1 / 2}=(250.0 \pm 0.2) \mathrm{GeV}, m_{0}=(100.0 \pm 0.2) \mathrm{GeV}, A_{0}=(-100 \pm 14) \mathrm{GeV}$ and $\tan \beta=10.0 \pm 0.4$.
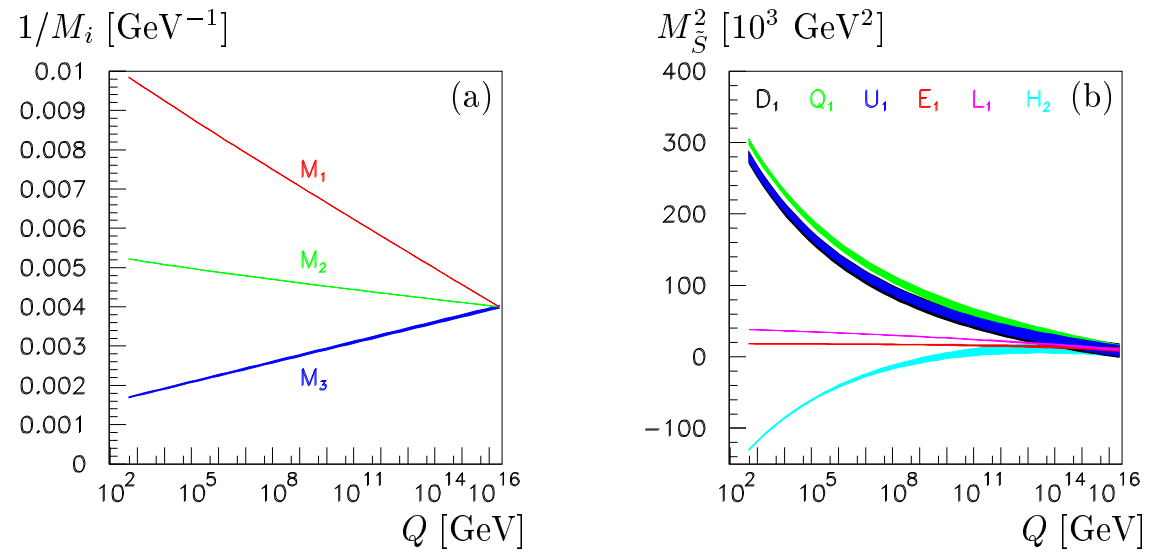

Figure 8. Evolution of (a) the gaugino mass parameters and (b) the first generation sfermion mass parameters from low to high scales $Q$ in the SPS1a scenario [33] for a coherent combination of LHC and linear collider analyses. From [43].

The phases $\phi_{M_{1}}$ and $\phi_{\mu}$ of $M_{1}$ and $\mu$ cause CP-violating effects already at tree-level. A useful tool to study these CP-violating effects are T-odd observables, based on triple products of momenta or spin vectors of the particles involved [34,44]. In [37] a T-odd asymmetry

$$
A_{T}=\frac{\int \operatorname{sign}\left\{\mathrm{O}_{\mathrm{T}}\right\}|\mathrm{T}|^{2} \text { dlips }}{\int|T|^{2} d \text { lips }}
$$


is defined for neutralino production and subsequent leptonic three-body decay with help of the triple product $O_{T}=\vec{p}_{\ell^{+}} \cdot\left(\vec{p}_{\ell^{-}} \times \vec{p}_{e^{-}}\right)$of the initial electron momentum $\vec{p}_{e^{-}}$and the two final lepton momenta $\vec{p}_{\ell^{+}}$and $\vec{p}_{\ell^{-}}$. Here $\int|T|^{2} d$ lips is proportional to the cross section $\sigma\left(e^{+} e^{-} \rightarrow \tilde{\chi}_{i}^{0} \tilde{\chi}_{j}^{0} \rightarrow \tilde{\chi}_{i}^{0} \tilde{\chi}_{k}^{0} \ell^{+} \ell^{-}\right)$. The asymmetry $A_{T}$ can be directly measured in the experiment without reconstruction of the momentum of the decaying neutralino or further final-state analyses. Analogous T-odd asymmetries in neutralino production and subsequent two-body decays have been studied in [35].

In figure 9 the T-odd asymmetry $A_{T}$ and the corresponding cross section at a linear collider with polarised beams is shown in a representative scenario of the unconstrained MSSM for the production $e^{+} e^{-} \rightarrow \tilde{\chi}_{1}^{0} \tilde{\chi}_{2}^{0}$ and the subsequent decay $\tilde{\chi}_{2}^{0} \rightarrow \tilde{\chi}_{1}^{0} \ell^{+} \ell^{-}$. For a centre of mass energy of $500 \mathrm{GeV}(350 \mathrm{GeV})$ the asymmetry reaches values $\left|A_{T}\right|=11 \%$ (13.5\%) for $\phi_{M_{1}}=0.2 \pi$ and $1.8 \pi$. A Monte Carlo study of $A_{T}$ including initial state radiation, beamstrahlung, SM backgrounds and detector effects has been given in [36]. It has been found that asymmetries $A_{T} \sim 10 \%$ are detectable after few years of running of a linear collider.
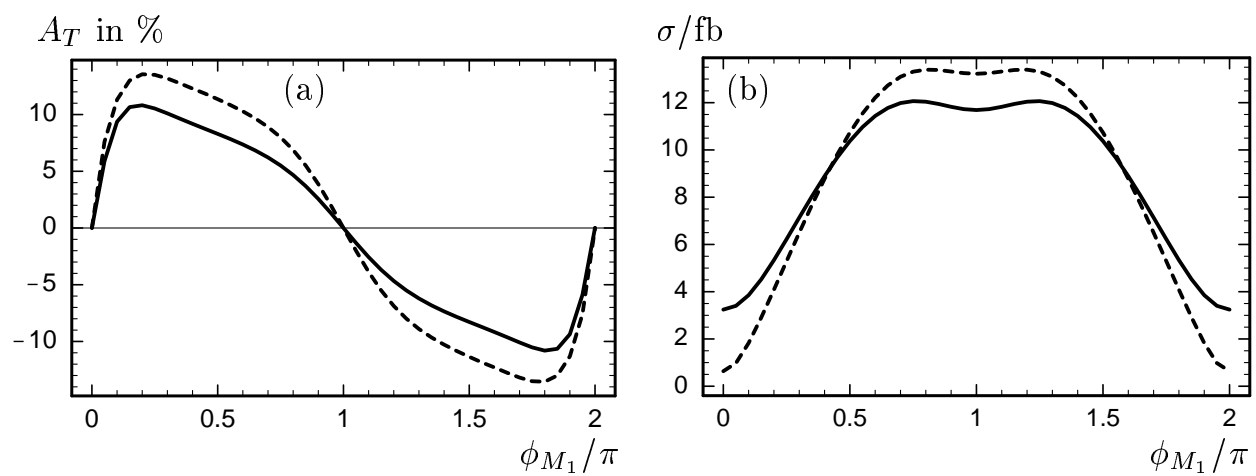

Figure 9. (a) $\mathrm{CP}$ asymmetry $A_{T}$ and (b) cross section $\sigma\left(e^{+} e^{-} \rightarrow \tilde{\chi}_{1}^{0} \tilde{\chi}_{2}^{0} \rightarrow\right.$ $\tilde{\chi}_{1}^{0} \tilde{\chi}_{1}^{0} \ell^{+} \ell^{-}$), summed over $\ell=e, \mu$, for $\left|M_{1}\right|=150 \mathrm{GeV}, M_{2}=300 \mathrm{GeV}$, $|\mu|=200 \mathrm{GeV}, \tan \beta=10, m_{\tilde{\ell}_{L}}=267.6 \mathrm{GeV}, m_{\tilde{\ell}_{R}}=224.4 \mathrm{GeV}$ and $\phi_{\mu}=0$ at a linear collider with beam polarisations $P_{e^{-}}=-0.8, P_{e^{+}}=+0.6$ and $\sqrt{s}=500 \mathrm{GeV}$ (solid), $\sqrt{s}=350 \mathrm{GeV}$ (dashed). From [37].

In the general SUSY Lagrangian R-parity can be violated by bilinear and trilinear couplings in the superpotential, which has two important consequences. On the one hand lepton number violating couplings contribute to the Majorana neutrino mass matrix (for a review see [45]), on the other hand the LSP is not stable and hence not necessarily a neutral particle. In sizeable regions of mSUGRA, GMSB or AMSB parameter space a charged slepton can be the LSP. Therefore, in $[46,47]$ the decay properties at colliders of such charged slepton LSPs are studied. This can be used to obtain information about the relative size of the bilinear and trilinear couplings and to decide which of them give the dominant contribution to the neutrino mass matrix.

In models, where neutrino oscillation properties are governed by bilinear couplings only [46], the slepton decay lengths are very different, $L\left(\tilde{\tau}_{1}\right) \ll L\left(\tilde{\mu}_{1}\right) \ll L\left(\tilde{e}_{1}\right)$, especially $L\left(\tilde{\tau}_{1}\right) / L\left(\tilde{\mu}_{1}\right) \sim m_{\mu}^{2} / m_{\tau}^{2}$. Furthermore ratios of branching ratios are strongly correlated with ratios of the bilinear couplings, hadronic final states are never visible and $B R\left(\tilde{e}_{1} \rightarrow\right.$ $\left.e \sum \nu_{i}\right) \approx 1$. 
In models with trilinear couplings contributing to the neutrino masses [47] the decay lengths of the charged slepton LSPs depend on the absolute values of the trilinear couplings and hence are strongly correlated with neutrino observables. Observing a finite decay length for $\tilde{e}_{1}$ or $\tilde{\mu}_{1}$ would imply that the corresponding trilinear couplings cannot contribute significantly to the neutrino masses. Contrarily, the decay length of $\tilde{\tau}_{1}$ in models, where the corresponding couplings determine the neutrino masses and where the solar neutrino mass squared difference is in the correct order of magnitude $5.1<\Delta m_{\odot}^{2} /\left(10^{-5} \mathrm{eV}^{2}\right)<19$, is just at the borderline of experimental accessibility. Furthermore also hadronic final states may have visible branching ratios and in models with only trilinear couplings contributing to the neutrino masses all right slepton LSPs obey $B R\left(\tilde{\ell}_{1} \rightarrow(e, \mu, \tau) \sum \nu_{i}\right)<0.5$.

\subsection{Extra dimensions}

A solution to the hierarchy problem can in principle be obtained by formulating gravity in $4+\delta$ dimensions, where $\delta=1,2,3, \ldots$ are the so-called "extra" dimensions, which are assumed to be compactified with a radius $R$. In the model of [48] it is assumed that SM physics is restricted to the 4-dimensional brane, whereas gravity acts in the $4+\delta$ dimensional bulk. In 4-dimensional space-time the Planck mass is $M_{\mathrm{Pl}}=1.2 \cdot 10^{19} \mathrm{GeV}$. In the $(4+\delta)$-dimensional space the corresponding Planck mass $M_{D}$ is given by $M_{D}^{2+\delta}=$ $M_{\mathrm{Pl}}^{2} / R^{\delta}$. Assuming further that the compactification radius $R$ is many orders of magnitude larger than the Planck length, $R \gg M_{\mathrm{Pl}}^{-1}, R$ and $\delta$ may be adjusted such that $M_{D} \approx$ $\mathcal{O}(1 \mathrm{TeV})$. In this way the Planck scale is close to the electroweak scale and there is no hierarchy problem $[48,49]$.

As a consequence of the compactification Kaluza-Klein towers of the gravitons can be excited. This leads to two possible signatures at an $e^{+} e^{-}$linear collider. The first one is $e^{+} e^{-} \rightarrow \gamma / Z+G_{n}$ where $G_{n}$ means the graviton and its Kaluza-Klein excitations, which appear as missing energy in the detector. The second signature is due to graviton exchange in $e^{+} e^{-} \rightarrow f \bar{f}$, which leads to a modification of cross sections and asymmetries compared to the SM prediction.

The cross section for $e^{+} e^{-} \rightarrow \gamma / Z+G$ has been calculated in [50]. The main background to this process is $e^{+} e^{-} \rightarrow \nu \bar{\nu} \gamma$, which strongly depends on the $e^{-}$beam polarisation. Table 2 from [3] shows the results on the sensitivity in $M_{D}$ for various values of $\delta$. Further aspects of "extra dimensions" physics can be found in [51,52].

Table 2. Sensitivity at $95 \% \mathrm{CL}$ in mass scale $M_{D}$ in $\mathrm{TeV}$ for direct graviton production in $e^{+} e^{-} \rightarrow \gamma G$ for various values of $\delta$ taking a $0.3 \%$ normalisation error. From [3].

\begin{tabular}{l|ccccc}
\hline$\delta$ & 2 & 3 & 4 & 5 & 6 \\
\hline$M_{D}$ for $\left(P_{e^{-}}, P_{e^{+}}\right)=(0,0)$ & 5.9 & 4.4 & 3.5 & 2.9 & 2.5 \\
$M_{D}$ for $\left(P_{e^{-}}, P_{e^{+}}\right)=(80 \%, 0)$ & 8.3 & 5.8 & 4.4 & 3.5 & 2.9 \\
$M_{D}$ for $\left(P_{e^{-}}, P_{e^{+}}\right)=(80 \%, 60 \%)$ & 10.4 & 6.9 & 5.1 & 4.0 & 3.3 \\
\hline
\end{tabular}




\section{Acknowledgements}

A.B. is grateful to the organisers of WHEPP-8 for their invitation and hospitality and for creating an inspiring atmosphere. This work was supported by the 'Fonds zur Förderung der wissenschaftlichen Forschung' of Austria, FWF Project No. P16592-N02 and by the European Community's Human Potential Programme under contract HPRN-CT-200000149.

\section{References}

[1] E. Accomando et al. [ECFA/DESY LC Physics Working Group Collaboration], Phys. Rept. 299 (1998) 1 [arXiv:hep-ph/9705442].

[2] T. Abe et al. [American Linear Collider Working Group Collaboration], in Proc. of the APS/DPF/DPB Summer Study on the Future of Particle Physics (Snowmass 2001) ed. N. Graf, arXiv:hep-ex/0106056.

[3] J. A. Aguilar-Saavedra et al. [ECFA/DESY LC Physics Working Group Collaboration], arXiv:hep-ph/0106315.

[4] K. Abe et al. [ACFA Linear Collider Working Group Collaboration], arXiv:hep-ph/0109166.

[5] G. Moortgat-Pick and H. M. Steiner, Eur. Phys. J. directC 3 (2001) 6 [arXiv:hep-ph/0106155].

[6] G. Moortgat-Pick et al. [POWER Collaboration], http://www.ippp.dur.ac.uk/ gudrid/power/.

[7] S. D. Rindani, "Role of transverse beam polarization in constraining new physics", talk at the International Conference on Linear Colliders, LCWS 2004, Paris, France, April 2004.

[8] Proceedings of the 4th International Workshop on Electron-Electron Interactions at TeV Energies, UC Santa Cruz, 2001, ed. C. A. Heusch, Int. J. Mod. Phys. A 18 (2003) 2733; R. M. Godbole, Nucl. Phys. Proc. Suppl. 126 (2004) 414 [arXiv:hep-ph/0311188].

[9] S. Heinemeyer and G. Weiglein, arXiv:hep-ph/0307177.

[10] The ALEPH, DELPHI, L3, OPAL, SLD Collaborations and the LEP Electroweak Working Group, arXiv:hep-ex/0312023.

[11] J. Erler, S. Heinemeyer, W. Hollik, G. Weiglein and P. M. Zerwas, Phys. Lett. B 486 (2000) 125 [arXiv:hep-ph/0005024];

S. Heinemeyer and G. Weiglein, arXiv:hep-ph/0012364;

J. Erler and S. Heinemeyer, in Proc. of the 5th International Symposium on Radiative Corrections (RADCOR 2000) ed. Howard E. Haber, arXiv:hep-ph/0102083.

[12] The CDF Collaboration, the D0 Collaboration, and the Tevatron Electroweak Working Group (P. Azzi et al.), arXiv:hep-ex/0404010;

V. M. Abazov et al. [D0 Collaboration], Nature 429 (2004) 638 [arXiv:hep-ex/0406031].

[13] Z. Hioki, "What will LC tell us on Top/QCD", talk at the International Conference on Linear Colliders, LCWS 2004, Paris, France, April 2004.

[14] S. Heinemeyer, S. Kraml, W. Porod and G. Weiglein, JHEP 0309 (2003) 075 [arXiv:hep$\mathrm{ph} / 0306181]$.

[15] M. Beneke et al., arXiv:hep-ph/0003033; in Standard model physics (and more) at the LHC, eds G. Altarelli and M. L. Mangano, CERN, Geneva, Switzerland, 2000 (CERN-2000-004) p. 419.

[16] A. H. Hoang, Phys. Rev. D 69 (2004) 034009 [arXiv:hep-ph/0307376]; Acta Phys. Polon. B 34 (2003) 4491 [arXiv:hep-ph/0310301].

[17] M. Martinez and R. Miquel, Eur. Phys. J. C 27 (2003) 49 [arXiv:hep-ph/0207315].

[18] A. Brandenburg, "News on Top Quark Physics and QCD", talk at the ECFA Workshop Physics and Detectors for a Linear Collider, Montpellier, France, Nov. 2003. 
[19] S. D. Rindani, Pramana 61 (2003) 33 [arXiv:hep-ph/0304046] and references therein.

[20] B. Grzadkowski and Z. Hioki, Phys. Rev. D 61 (2000) 014013 [arXiv:hep-ph/9805318]; Phys. Lett. B 529, 82 (2002) [arXiv:hep-ph/0112361]; Phys. Lett. B 557, 55 (2003) [arXiv:hep$\mathrm{ph} / 0208079]$.

[21] K. Hagiwara, R. D. Peccei, D. Zeppenfeld and K. Hikasa, Nucl. Phys. B 282 (1987) 253.

[22] M. Diehl, O. Nachtmann and F. Nagel, Eur. Phys. J. C 32 (2003) 17 [arXiv:hep-ph/0306247].

[23] I. Bozovic-Jelisavcic, K. Mönig and J. Sekaric, arXiv:hep-ph/0210308;

K. Mönig and J. Sekaric, LC-PHSM-2003-072.

[24] A. Djouadi, Pramana 60 (2003) 215 [arXiv:hep-ph/0205248].

[25] C. Castanier, P. Gay, P. Lutz and J. Orloff, arXiv:hep-ex/0101028;

M. Battaglia, E. Boos and W. M. Yao, in Proc. of the APS/DPF/DPB Summer Study on the Future of Particle Physics (Snowmass 2001) ed. N. Graf, eConf C010630 (2001) E3016 [arXiv:hep-ph/0111276];

U. Baur, T. Plehn and D. L. Rainwater, Phys. Rev. D 68 (2003) 033001 [arXiv:hep-ph/0304015].

[26] A. Djouadi, W. Kilian, M. Mühlleitner and P. M. Zerwas, Eur. Phys. J. C 10 (1999) 27 [arXiv:hep-ph/9903229].

[27] B. C. Allanach, A. Djouadi, J. L. Kneur, W. Porod and P. Slavich, arXiv:hep-ph/0406166.

[28] A. Pilaftsis, Phys. Lett. B 435 (1998) 88 [arXiv:hep-ph/9805373];

A. Pilaftsis and C. E. M. Wagner, Nucl. Phys. B 553 (1999) 3 [arXiv:hep-ph/9902371];

S. Y. Choi, M. Drees and J. S. Lee, Phys. Lett. B 481 (2000) 57 [arXiv:hep-ph/0002287];

M. Carena, J. R. Ellis, A. Pilaftsis and C. E. M. Wagner, Nucl. Phys. B 586 (2000) 92 [arXiv:hep-ph/0003180]; Phys. Lett. B 495 (2000) 155 [arXiv:hep-ph/0009212]; Nucl. Phys. B 625 (2002) 345 [arXiv:hep-ph/0111245];

G. L. Kane and L. T. Wang, Phys. Lett. B 488 (2000) 383 [arXiv:hep-ph/0003198];

S. Heinemeyer, Eur. Phys. J. C 22 (2001) 521 [arXiv:hep-ph/0108059];

M. Carena, J. R. Ellis, S. Mrenna, A. Pilaftsis and C. E. M. Wagner, Nucl. Phys. B 659 (2003) 145 [arXiv:hep-ph/0211467];

M. Frank, S. Heinemeyer, W. Hollik and G. Weiglein, arXiv:hep-ph/0212037;

J. S. Lee, A. Pilaftsis, M. Carena, S. Y. Choi, M. Drees, J. R. Ellis and C. E. M. Wagner, Comput. Phys. Commun. 156 (2004) 283 [arXiv:hep-ph/0307377].

[29] A. Bartl, H. Fraas and W. Majerotto, Nucl. Phys. B 278 (1986) 1.

[30] J. L. Kneur and G. Moultaka, Phys. Rev. D 61 (2000) 095003 [arXiv:hep-ph/9907360];

V. Barger, T. Han, T. J. Li and T. Plehn, Phys. Lett. B 475 (2000) 342 [arXiv:hep-ph/9907425]; S. Y. Choi, A. Djouadi, M. Guchait, J. Kalinowski, H. S. Song and P. M. Zerwas, Eur. Phys. J. C 14 (2000) 535 [arXiv:hep-ph/0002033];

S. Y. Choi, J. Kalinowski, G. Moortgat-Pick and P. M. Zerwas, Eur. Phys. J. C 22 (2001) 563 [Addendum-ibid. C 23 (2002) 769] [arXiv:hep-ph/0108117];

G. J. Gounaris and C. Le Mouël, Phys. Rev. D 66 (2002) 055007 [arXiv:hep-ph/0204152].

[31] G. Moortgat-Pick, H. Fraas, A. Bartl and W. Majerotto, Eur. Phys. J. C 9, 521 (1999) [Erratumibid. C 9, 549 (1999)] [arXiv:hep-ph/9903220].

[32] S. Kawasaki, T. Shirafuji, S. Y. Tsai, Prog. Theor. Phys. 49 (1973) 1656;

D. A. Dicus, E. C. G. Sudarshan and X. Tata, Phys. Lett. B 154 (1985) 79.

[33] N. Ghodbane and H. U. Martyn, arXiv:hep-ph/0201233;

B. C. Allanach et al., Eur. Phys. J. C 25 (2002) 113 [arXiv:hep-ph/0202233].

[34] S. Y. Choi, H. S. Song and W. Y. Song, Phys. Rev. D 61 (2000) 075004 [arXiv:hep-ph/9907474].

[35] A. Bartl, H. Fraas, O. Kittel and W. Majerotto, Phys. Rev. D 69 (2004) 035007 [arXiv:hep$\mathrm{ph} / 0308141]$;

A. Bartl, H. Fraas, O. Kittel and W. Majerotto, arXiv:hep-ph/0402016.

[36] J. A. Aguilar-Saavedra, arXiv:hep-ph/0404104.

[37] A. Bartl, H. Fraas, S. Hesselbach, K. Hohenwarter-Sodek and G. Moortgat-Pick, arXiv:hep- 
$\mathrm{ph} / 0406190$.

[38] H. U. M. Martyn, arXiv:hep-ph/0406123.

[39] A. Bartl, H. Eberl, S. Kraml, W. Majerotto and W. Porod, Eur. Phys. J. directC 2 (2000) 6 [arXiv:hep-ph/0002115].

[40] A. Bartl, K. Hidaka, T. Kernreiter and W. Porod, Phys. Lett. B 538 (2002) 137 [arXiv:hepph/0204071]; Phys. Rev. D 66 (2002) 115009 [arXiv:hep-ph/0207186].

[41] A. Bartl, S. Hesselbach, K. Hidaka, T. Kernreiter and W. Porod, arXiv:hep-ph/0306281; Phys. Lett. B 573 (2003) 153 [arXiv:hep-ph/0307317]; arXiv:hep-ph/0311338.

[42] G. A. Blair, W. Porod and P. M. Zerwas, Eur. Phys. J. C 27 (2003) 263 [arXiv:hep-ph/0210058].

[43] B. C. Allanach, G. A. Blair, S. Kraml, H. U. Martyn, G. Polesello, W. Porod and P. M. Zerwas, arXiv:hep-ph/0403133.

[44] J. F. Donoghue, Phys. Rev. D 18 (1978) 1632;

Y. Kizukuri and N. Oshimo, Phys. Lett. B 249 (1990) 449;

G. Valencia, arXiv:hep-ph/9411441.

[45] B. Mukhopadhyaya, arXiv:hep-ph/0301278.

[46] M. Hirsch, W. Porod, J. C. Romao and J. W. F. Valle, Phys. Rev. D 66 (2002) 095006 [arXiv:hep-ph/0207334].

[47] A. Bartl, M. Hirsch, T. Kernreiter, W. Porod and J. W. F. Valle, JHEP 0311 (2003) 005 [arXiv:hep-ph/0306071].

[48] N. Arkani-Hamed, S. Dimopoulos and G. R. Dvali, Phys. Lett. B 429 (1998) 263 [arXiv:hepph/9803315]; Phys. Rev. D 59 (1999) 086004 [arXiv:hep-ph/9807344];

I. Antoniadis, N. Arkani-Hamed, S. Dimopoulos and G. R. Dvali, Phys. Lett. B 436 (1998) 257 [arXiv:hep-ph/9804398].

[49] I. Antoniadis, Phys. Lett. B 246 (1990) 377;

I. Antoniadis, C. Munoz and M. Quiros, Nucl. Phys. B 397 (1993) 515 [arXiv:hep-ph/9211309];

I. Antoniadis and K. Benakli, Phys. Lett. B 326 (1994) 69 [arXiv:hep-th/9310151];

I. Antoniadis, K. Benakli and M. Quiros, Phys. Lett. B 331 (1994) 313 [arXiv:hep-ph/9403290].

[50] G. F. Giudice, R. Rattazzi and J. D. Wells, Nucl. Phys. B 544 (1999) 3 [arXiv:hep-ph/9811291].

[51] C. Burgess, these Proceedings;

G. Landsberg, these Proceedings.

[52] T. G. Rizzo, in Proc. of the APS/DPF/DPB Summer Study on the Future of Particle Physics (Snowmass 2001) ed. N. Graf, eConf C010630 (2001) P301 [arXiv:hep-ph/0108235];

J. Hewett and M. Spiropulu, Ann. Rev. Nucl. Part. Sci. 52 (2002) 397 [arXiv:hep-ph/0205106]. 